\title{
Battarrea stevenii (Libosch.) Fr. (Tulostomataceae), um raro fungo xerófilo: primeiro registro para o Brasil ${ }^{1}$
}

\author{
Bianca Denise Barbosa Silva²,3, Anileide Gomes Leite ${ }^{2}$ e Iuri Goulart Baseia ${ }^{2}$
}

Recebido em 6/03/2006. Aceito em 6/12/2006

\begin{abstract}
RESUMO - (Battarrea stevenii (Libosch.) Fr. (Tulostomataceae), um raro fungo xerófilo: primeiro registro para o Brasil). Battarrea stevenii, uma rara espécie ocorrente em regiões áridas, é descrita macro- e microscopicamente pela primeira vez para o Brasil e comparada com a espécie similar Battarrea phalloides.
\end{abstract}

Palavras-chave: Gasteromycetes, Agaricales, Tulostomataceae, neotrópicos, taxonomia

\begin{abstract}
Battarrea stevenii (Libosch.) Fr. (Tulostomataceae), a rare xerophilous fungi: first record from Brazil). Battarrea stevenii, a rare species from xeric areas, is described macro- and microscopically for the first time from Brazil, and compared to the closest species, Battarrea phalloides.
\end{abstract}

Key words: Gasteromycetes, Agaricales, Tulostomataceae, Neotropics, taxonomy

\section{Introdução}

O gênero Battarrea foi criado por Persoon em 1801 e, segundo Kreisel (2001), compreende duas espécies: B. phalloides (Dicks.) Pers. e B. stevenii (Libosch.) Fr., caracterizadas por apresentar, na maturidade, um esporóforo hemisférico com deiscência circular, apoiado em um estipe escamoso, bem como elatérios na gleba (Miller Junior \& Miller 1988).

Este grupo apresenta preferências por habitat xerófilos e arenosos, com chuvas escassas (Cunningham 1944; Bottomley 1948; Pilát 1970; Miller Junior \& Miller 1988; Nieves-Rivera et al. 1998; Jacobson et al. 1999). Até o momento, há apenas o registro de Battarrea phalloides para o Brasil feito por Rick (1961) e Sobestiansky (2005) para o Rio Grande do Sul.

O presente estudo constitui parte dos estudos referentes à micobiota no semi-árido brasileiro, região com vegetação de caatinga e índices pluviométricos muito baixos, determinando extensos períodos de secas (Leal et al. 2004). O conhecimento sobre a diversidade de fungos nessa região ainda é escasso, sobretudo sobre os fungos gasteróides. Este estudo visa contribuir para maior conhecimento sobre a diversidade micológica no semi-árido nordestino.

\section{Material e métodos}

O espécime foi coletado no período chuvoso, em janeiro de 2003, na Reserva Biológica de Serra Negra $\left(8^{\circ} 38^{\prime}-8^{\circ} 35^{\prime} \mathrm{S}\right.$ e $\left.38^{\circ} 02^{\prime}-38^{\circ} 04^{\prime} \mathrm{W}\right)$, localizada nos municípios de Floresta, Inajá e Tacaratu, sertão do Estado de Pernambuco, Brasil. A reserva abrange uma área total de 1.100 ha, sendo composta por Floresta Tropical Úmida e principalmente por vegetação de Caatinga.

As análises macroscópicas foram realizadas com o material seco e baseadas na literatura sobre Gasteromycetes (Bottomley 1948; Miller Junior \& Miller 1988). Foram observados a dimensão do perídio, a natureza e a organização da gleba, a consistência e o tamanho do estipe. Para os caracteres microscópicos foram montadas porções da gleba em $\mathrm{KOH}$ a $3 \%$ para análises dos esporos e elatérios e em reagente azul de algodão para

\footnotetext{
Parte da Dissertação de Mestrado da primeira Autora

2 Universidade Federal do Rio Grande do Norte, CB, Departamento de Botânica, Ecologia e Zoologia, Laboratório de Micologia, Campus Universitário, Lagoa Nova, 59072-970 Natal, RN, Brasil (baseia@cb.ufrn.br)

3 Autor para correspondência: biancadeni@yahoo.com.br
} 
observações de paracapilícios. Adicionalmente, foram feitas observações dos esporos e elatérios em Microscópio Eletrônico de Varredura (MEV) modelo Shimadzu SSX-550. O material examinado foi depositado no Herbário da UFRN. A coloração segue Kornerup \& Wanscher (1978).

\section{Resultados}

Chave para identificação das espécies de Battarrea

1. Basidioma até $20 \mathrm{~cm}$ de altura; volva gelatinosa B. phalloides

1. Basidioma maior que $20 \mathrm{~cm}$ de altura; volva membranosa

B. stevenii

1. Battarrea stevenii (Libosch) Fr., Syst. Mycol. 3: 7, 1832

Fig. 1-2

Basiônimo: Dendromyces stevenii Libosch. 1814, Saccardo's Syll. Fung. VII: 66; XV: 112

= Sphaericeps lignipes Welw \& Currey, Trans. Linn. Soc. London 26: 290, 1868

= Battarrea guicciardiniana Cesati, Atti Accad. Sci. Fis. 7: 1, 1875 (sinônimos seguem Kreisel 2001).

Basidioma 10,2 cm alt. Esporóforo subgloboso depresso, 2,0-2,5×1,8-2,0 cm, deiscência circular. Porção superior do endoperídio caduco expondo a gleba, branca amarelada (KW-4A2). Porção inferior do endoperídio lisa, papirácea, firmemente presa ao estipe, branca amarelada (KW-4A2). Estipe $9,5 \mathrm{~cm}$ alt., lenhoso, escamoso-fibroso, marrom claro (KW-6D5), levemente delgado no ápice. Gleba

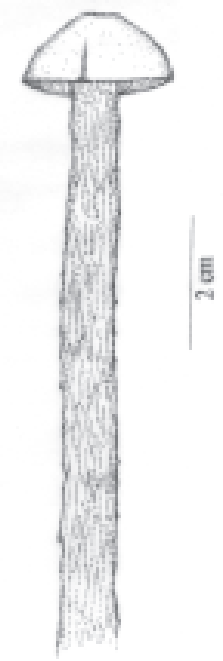

Figura 1. Battarrea stevenii (Libosch.) Fr.: basidioma maduro.
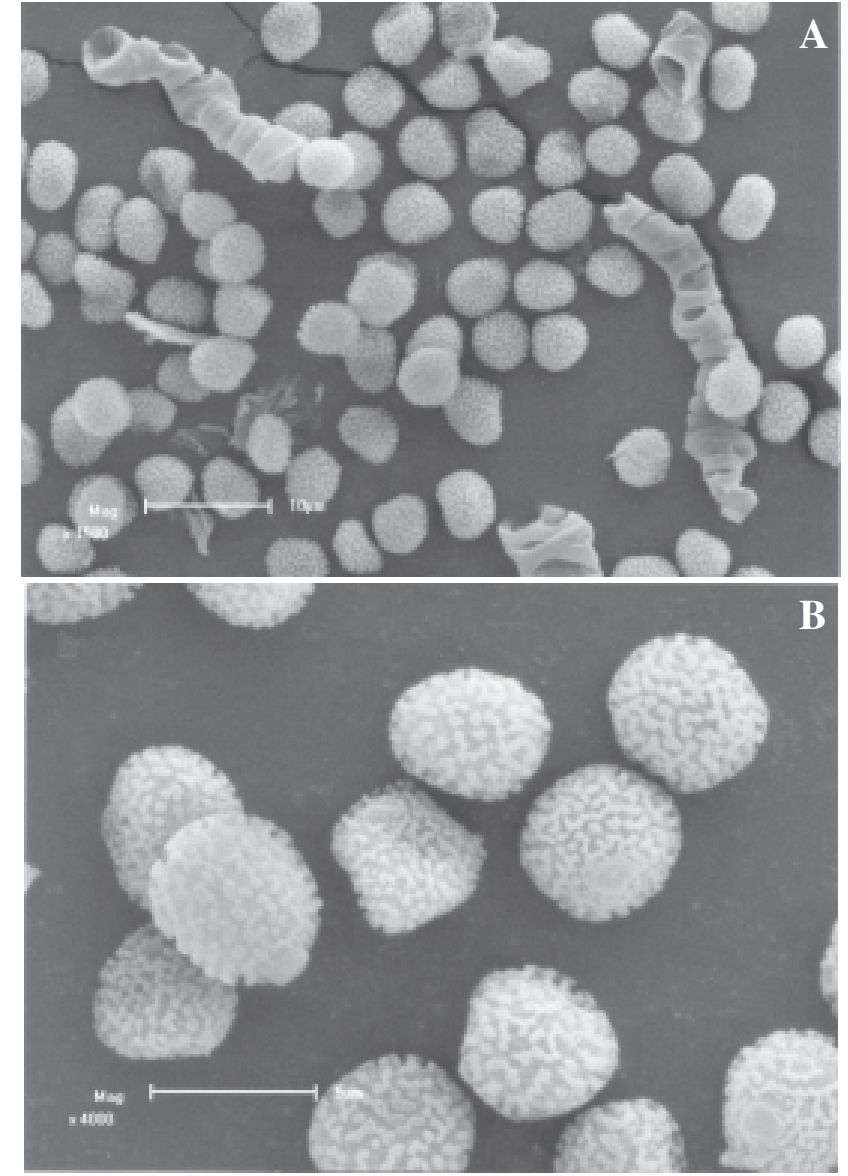

Figura 2. Battarrea stevenii (Libosch.) Fr. A. Basidiósporos. B. Basidiósporos e elatérios.

pulverulenta, marrom (KW-6E8), cobrindo a porção inferior do endoperídio. Paracapilícios presentes, 2,0-6,0 $\mu \mathrm{m}$ diâm., hialinos, septados. Elatérios 3,9-8,0×4,2-9,0 $\mu \mathrm{m}$, levemente amarelados, espiralados. Basidiósporos globosos a subglobosos, 5,0-6,5x 4,0-5,5 um diâm., marrom amarelados, levemente verrugosos. A gleba exala odor similar a bacalhau.

Material estudado: BRASIL. Pernambuco: Reserva Biológica de Serra Negra, 16/I/2003, Baseia 1100 (UFRN-Fungos 212).

Habitat: Basidioma solitário crescendo sobre solo arenoso.

Distribuição: Estados Unidos (Rea 1942), México (Moreno et al. 1995), Porto Rico (Nieves-Rivera et al. 1998), Namíbia (Jacobson et al. 1999), Espanha (Calonge \& Palacios 2000).

\section{Discussão}

O gênero Battarrea é constituído por apenas duas espécies, B. phalloides e B. stevenii (Kreisel 2001). 
As principais características distintivas são: dimensão dos basidiomas, maiores em $B$. stevenii, e consistência da volva, gelatinosa em B. phalloides (Maublanc \& Malençon 1930; Watling et al. 1995; Moreno et al. 1995; Calonge 1996; 1998; Jacobson et al. 1999; Calonge \& Palacios 2000). Por outro lado, alguns autores consideram-nas uma só espécie, devido à presença de similaridades microestruturais, tais como ornamentação dos esporos e elatérios (Rea 1942; Martin \& Johannesson 2000; Jeffries \& McLain 2004). Segundo Jeffries \& McLain (2004), as características diferenciais entre as espécies podem ser influenciadas pelas condições climáticas. Kreisel (2001) publicou uma extensa lista de Gasteromycetes na qual concorda com as divergências morfológicas entre estas duas espécies que, embora pequenas, dão suporte suficiente para que sejam tratadas com distinção. Diante do exposto, tornam-se importantes estudos adicionais do holótipo, como proposto por Moreno et al. (1995) e NievesRivera et al. (1998). No entanto, este não foi localizado.

\section{Agradecimentos}

Os autores agradecem ao Centro de Tecnologias do Gás do Rio Grande do Norte (CTGÁS) por disponibilizar o Microscópio Eletrônico de Varredura e a Tereza Cristina de Oliveira Galvão, pela ilustração.

\section{Referências bibliográficas}

Bottomley, A.M. 1948. Gasteromycetes of South Africa. Bothalia 4: 473-810.

Calonge, F.D. 1996. Some aspects on the ecology and taxonomy of the Iberian Peninsula (Spain and Portugal) Gasteromycetes. Micologia e Vegetazione Mediterranea 11: 115-128.

Calonge, F.D. 1998. Gasteromycetes, I. Lycoperdales, Nidulariales, Phallales, Sclerodermatales, Tulostomatales. Flora Mycologica Iberica 25: 1-271.

Calonge, F.D. \& Palacios, D. 2000. Novedades de Gasteromycetes para Navarra. Boletín de la Sociedad Micológica de Madrid 25: 307-308.

Versão eletrônica do artigo em www.scielo.br/abb
Cunningham, G.H. 1944. The Gasteromycetes of Australia and New Zealand. New Zealand, Dunedin.

Jeffries, P. \& McLain, L. 2004. Synonymy between Battarrea phalloides and B. stevenii. English Nature Research Reports 625: 9-32.

Jacobson, K.M.; Jacobson, P.J. \& Miller Junior, O.K. 1999. The autecology of Battarrea stevenii in ephemeral rivers of southwestern Africa. Mycological Research 103: 9-17.

Kornerup, A. \& Wanscher, J.H. 1978. Methuen Handbook of Colour. $3^{\text {th }}$ ed., London, Eyre Methuen.

Kreisel, H. 2001. Checklist of the gasteral and secotioid Basidiomycetes of Europe, Africa, and the Middle East. Österreichische Zeitschrift für Pilzkunde 10: 213-313.

Leal, I.R.; Tabarelli, M. \& Silva, J.M.C. 2004. Ecologia e Conservação da Caatinga: Uma Introdução ao Desafio. Recife, EDUFPE.

Martin, M.P. \& Johannesson, H. 2000. Battarrea phalloides and $B$. stevenii, insight into a long-standing taxonomic puzzle. Mycotaxon 74: 67-75.

Maublanc, M.A. \& Malençon, G.J.L. 1930. Recherches sur le Battarraea guicciardiniana Ces. Bulletin Trimestriel de la Société Mycologique de France 46: 43-73.

Miller Junior, O.K. \& Miller, H.H. 1988. Gasteromycetes: Morphology and Developmental Features. Eureka, Mad River.

Moreno, G.; Altés, A.; Ochoa, C. \& Wright, J.E. 1995. Contribution to the study of the Tulostomataceae in Baja California, Mexico. I. Mycologia 87: 96-120.

Nieves-Rivera, A.M.; Lodge, D.J. \& Miller Junior, O.K. 1998. Contributions to the study of Gasteromycetes of Puerto Rico. Journal of American Amateur Mycology 13: $50-58$.

Pilát, A. 1970. Uber Battarrea stevenii (Lib.) Fr. Zeitschrift für Pilzkunde 36: 2.

Rea, P.M. 1942. Fungi of southern California. I. Mycologia 34: 563-574.

Rick, J. 1961. Basidiomycetes Eubasidii no Rio Grande do Sul. Brasília. Iheringia 9: 451-480.

Sobestiansky, G. 2005. Contribution to a macromycete survey of the States of Rio Grande do Sul and Santa Catarina in Brazil. Brazilian Archives of Biology and Technology 48: 437-457.

Watling, R.; Gucin, F. \& Isiloglu, M.G. 1995. Battarrea phalloides - its history, biology and extension to its distribution. Nova Hedwigia 60: 13-18. 